\title{
The surface carbon and nitrogen abundances in models of ultra metal-poor stars
}

\author{
H. Schlattl ${ }^{1}$, M. Salaris ${ }^{1}$, S. Cassisi ${ }^{2}$, and A. Weiss ${ }^{3}$ \\ 1 Astrophysics Research Institute, Liverpool John Moores University, Twelve Quays House, Egerton Wharf, \\ Birkenhead CH41 1LD, UK \\ 2 INAF - Osservatorio Astronomico Collurania, via Mentore Maggini, 64100 Teramo, Italy \\ 3 Max-Planck-Institut für Astrophysik, Karl-Schwarzschild-Str. 1, 85741 Garching, Germany
}

Received 16 April 2002 / Accepted 28 May 2002

\begin{abstract}
We investigate whether the observed high number of carbon- and nitrogen-enhanced extremely metal-poor stars could be explained by peculiar evolutionary properties during the core He flash at the tip of the red giant branch. For this purpose we compute a series of detailed stellar models expanding upon our previous work; in particular, we investigate if during the major He flash the penetration of the helium convective zone into the overlying hydrogen-rich layers can produce carbonand nitrogen-rich abundances in agreement with current spectroscopic observations. The dependence of this phenomenon on selected model input parameters, such as initial metallicity and treatment of convection is examined in detail.
\end{abstract}

Key words. stars: abundances - stars: evolution - stars: interiors - stars: late type

\section{Introduction}

Spectroscopic observations of ultra metal-poor (UMP) stars have disclosed that carbon and nitrogen are significantly overabundant with respect to Fe, compared to typical halo stars which show $[\mathrm{C} / \mathrm{Fe}] \approx[\mathrm{N} / \mathrm{Fe}] \approx 0.0$. According to Beers (1999) and Rossi et al. (1999) about $10 \%$ of the stars with $[\mathrm{Fe} / \mathrm{H}] \leq-2.5$ display $[\mathrm{C} / \mathrm{Fe}] \geq 1.0$; this fraction rises to about $25 \%$ when $[\mathrm{Fe} / \mathrm{H}] \leq-3.0$. Also $\mathrm{N}$ appears to be overabundant by the same amount, while $\alpha$-elements show a ratio with respect to $\mathrm{Fe}$ that is typical of halo stars, i.e. $[\alpha / \mathrm{Fe}] \approx 0.4$. As discussed, e.g., by Norris et al. (1997a), it is difficult to explain these abundance ratios in terms of binary star evolution or ejecta from very massive Population III supernovae, which in particular produce few nitrogen but vast amounts of oxygen (Heger \& Woosley 2002), so that the origin of the surface abundances of UMP stars is still shrouded in mystery.

Concerning the nature of the UMPs, different ideas may be followed, the first one being that they are true Pop III stars i.e. of initially zero metallicity - with the observed heavy elements (Fe, etc.) resulting from pollution of just the envelope by other stars such as supernovae. In two recent papers (Weiss et al. 2000, Paper I; and Schlattl et al. 2001, Paper II) we have thus discussed in detail the evolution of low-mass zero-metal stars, including the effect of atomic diffusion, mass loss and surface metal pollution. One important result was that the small entropy barrier between the $\mathrm{H}$ - and $\mathrm{He}$-rich regions allows the

Send offprint requests to: $\mathrm{H}$. Schlattl, e-mail: hs@astro.livjm.ac.uk
He-flash driven convective zone to penetrate the overlying $\mathrm{H}$ rich layers at the tip of the red giant branch. The consequent inward migration of protons into high-temperature regions leads to an H-shell flash, which further increases the extension of the central convective region. At the late stages of this phase the convective envelope deepens and merges with the inner convective zone. The initially metal-free surface is therefore enriched by a large amount of matter processed in $\mathrm{He}$ - and $\mathrm{H}$-burning reactions. In particular, very high $\mathrm{C}$ and $\mathrm{N}$ abundances follow. We denote this process of surface $\mathrm{C}$ and $\mathrm{N}$ enrichment as HElium Flash induced Mixing (HEFM).

This scenario - already suggested by Fujimoto et al. (2000) - could potentially explain the anomalous abundance pattern at the surface of UMP stars, since via this mechanism high abundances of $\mathrm{C}$ and $\mathrm{N}$ are produced in the post He-flash phases. In Paper II we have shown that an initially metal-free star of $0.82 M_{\odot}$, polluted by $0.0003 M_{\odot}$ of $Z=0.02$ material, undergoes HEFM and reproduces in its post He-flash phase approximately luminosity and surface gravity of two of the best studied UMP stars, namely CS 22892-052 and CS 22957027. The stellar mass was chosen such that the age on the RGB of 13.7 Gyr is compatible with the current estimates of the age of the universe, and with the age of CS 22892-052 estimated by Cowan et al. (1997) from nuclear chronology $(15.6 \pm 4.6 \mathrm{Gyr})$. The amount and composition of the polluting material was adjusted such that the observed surface $[\mathrm{Fe} / \mathrm{H}]$ abundance $([\mathrm{Fe} / \mathrm{H}] \approx-3)$ was obtained and, as the result of the HEFM, $[\mathrm{C} / \mathrm{Fe}]$ and $[\mathrm{N} / \mathrm{Fe}]$ ratios considerably larger than zero resulted. However, the values of $[\mathrm{C} / \mathrm{Fe}]$ and $[\mathrm{N} / \mathrm{Fe}]$ predicted 
Table 1. Data for the UMP stars discussed in the text. The columns show star name, logarithm of the surface gravity, effective temperature, $[\mathrm{Fe} / \mathrm{H}],[\mathrm{C} / \mathrm{Fe}],[\mathrm{N} / \mathrm{Fe}]$, and ${ }^{12} \mathrm{C} /{ }^{13} \mathrm{C}$ ratio, respectively. The errors on $T_{\text {eff }}$ and $\log g$ of $\mathrm{CS} 22892-052$ have been estimated by us.

\begin{tabular}{|c|c|c|c|c|c|c|}
\hline star & $\log g$ & $T_{\text {eff }}$ & {$[\mathrm{Fe} / \mathrm{H}]$} & {$[\mathrm{C} / \mathrm{Fe}]$} & {$[\mathrm{N} / \mathrm{Fe}]$} & ${ }^{12} \mathrm{C} /{ }^{13} \mathrm{C}$ \\
\hline $\mathrm{CS} 22892-052^{a}$ & $1.5 \pm 0.5$ & $4850 \pm 100$ & $-2.97 \pm 0.20$ & $1.10 \pm 0.23$ & $1.0 \pm 0.52$ & $\gtrsim 10$ \\
\hline CS $22957-027^{b}$ & $2.25 \pm 1.0$ & $4839 \pm 130$ & $-3.43 \pm 0.12$ & $2.20 \pm 0.30$ & $2.0 \pm 0.50$ & $\approx 10$ \\
\hline CS $22948-027^{c}$ & $1.0 \pm 0.3$ & $4600 \pm 100$ & $-2.57 \pm 0.23$ & $2.0 \pm 0.18$ & $1.8 \pm 0.24$ & $\approx 10$ \\
\hline CS $22949-037^{d}$ & $1.7 \pm 0.3$ & $4900 \pm 100$ & $-3.79 \pm 0.16$ & $1.05 \pm 0.20$ & $2.7 \pm 0.40$ & - \\
\hline
\end{tabular}

${ }^{a}$ Data from Norris et al. (1997a).

${ }^{b}$ C \& N abundances from Norris et al. (1997b), [Fe/H], $g$, and $T_{\text {eff }}$ from Bonifacio et al. (1998).

$c$ Data from Aoki et al. (2002).

${ }^{d}$ Data from Norris et al. (2001).

by the models are about 2 orders of magnitude higher than the observed ones; i.e., we predict $[\mathrm{C} / \mathrm{Fe}] \approx[\mathrm{N} / \mathrm{Fe}] \approx 4$, while observations of these stars yield $[\mathrm{C} / \mathrm{Fe}] \approx[\mathrm{N} / \mathrm{Fe}] \approx 1-2$ (see Table 1). We note, however, that higher $\mathrm{N}$ overabundances are observed, e.g., in CS 22949-037 (Norris et al. 2001).

An alternative explanation for the $\mathrm{C}$ and $\mathrm{N}$ enhancement in UMPs is provided by HEFM during the thermally pulsating asymptotic giant-branch phase (TP-AGB) which occurs in metal-free stars with $M \gtrsim 1 M_{\odot}$ (Cassisi et al. 1996; Fujimoto et al. 2000). However, age, surface gravity, and effective temperature of these AGB stars are more difficult to reconcile with those of the observed objects, which are therefore unlikely to be TP-AGB stars themselves. The idea in this case is that the observed low-mass UMP objects form binary systems with more massive AGB stars, which have transferred part of their C- and N-enriched envelope to their companion (Fujimoto et al. 2000). A potential problem of this hypothesis is that HEFM during the TP-AGB of intermediate-mass stars may enhance the surface oxygen content similar to carbon. This feature can be found in models of Siess et al. (2002), which however do not agree in this respect with computations of Chieffi et al. (2001). An oxygen enhancement of $[\mathrm{O} / \mathrm{Fe}] \approx 1-2$ might not necessarily be in contradiction to observations, as only very weak upper limits on the $[\mathrm{O} / \mathrm{Fe}]$ ratio exist (Sneden et al. 1996; Norris et al. 1997a). But the main problem of this scenario is that not all UMPs are in binary systems, and the orbital periods of those who are, are inconsistent with an AGB mass transfer paradigm (Preston \& Sneden 2001). Moreover, the origin of Fe in these stars is as uncertain as in our favoured scenario. It is presently not clear whether intermediate-mass stars with $[\mathrm{Fe} / \mathrm{H}] \approx-3$ undergo HEFM during the TP-AGB or rather evolve like ordinary Pop II objects. In the latter case the surface $\mathrm{C}, \mathrm{N}, \mathrm{O}$, and Fe abundances of UMP stars would then be the result of mass transfer from their companion and the accretion of further material, e.g., a nearby supernova.

In summary, HEFM during the major He flash is presently the most promising astrophysical explanation for the high [C, N/Fe] ratios observed in UMP stars despite their failure of reproducing the absolute value of the $\mathrm{C}$ and $\mathrm{N}$ enrichment. Therefore we are studying to what extend HEFM occurs also in low-mass stars with initial $Z>0$, and whether those objects are showing the observed $[\mathrm{C}, \mathrm{N} / \mathrm{Fe}]$ ratios of CS 22892-052 and CS 22957-027. This extension of our model assumption is warranted by the results about star formation in primordial environments, which agree on the fact that the formation of (very) massive objects is strongly favoured in the absence of metals (Abel et al. 2002; Bromm et al. 2002). If this indeed is the case, the UMPs would constitute the extreme end of Pop II, and had formed out of material already bearing the signature of individual supernova events (Shigeyama \& Tsujimoto 1998).

The final alternative for the origin of the carbon-rich UMPs is that they were already born with the abundances they now show. This scenario would receive support if single dwarfs of similar composition should definitely be identified. While one shifts the problem of identifying the source of the peculiar abundances to other stars, it is worthwhile, nevertheless, to investigate the evolution of such $\mathrm{C}$-rich stars. For example, they are expected to reach standard RGB-tip luminosities and low gravities, which could be compared with those of observed objects. Stars of extremely low metallicity ( $Z=0$ in particular), have a definitely shorter RGB, in contrast.

In addition to the three plausible models for the origin of the C-rich UMPs, we also investigate in this paper a physical problem associated with the HEFM. Due to the large uncertainties still affecting the treatment of convection in stellar models, we will assess the sensitivity of the HEFM process to different assumptions about the extension and mixing efficiency of convective regions in the stellar interiors.

The paper is structured as follows. Section 2 summarizes briefly the model computational techniques, input physics, and evolution of low-mass stars undergoing HEFM. Section 3 discusses the occurrence of HEFM and resulting surface abundances for different initial metallicities and various parameterizations of the stellar convective regions; Sect. 4 summarizes the results.

\section{Modelling and evolution of stars undergoing HEFM during the He flash}

All calculations (as the ones in Paper II) have been performed using the Garching stellar evolution code (Weiss \& Schlattl 2000). The numerical details are presented in Paper II and will not be repeated here. The important feature of our code is the ability to follow in detail the evolution through the He flash considering simultaneously mixing and burning processes. Mixing is described by means of a time-dependent algorithm that treats convection as a fast diffusive process, where the diffusion constant is proportional to the convective 


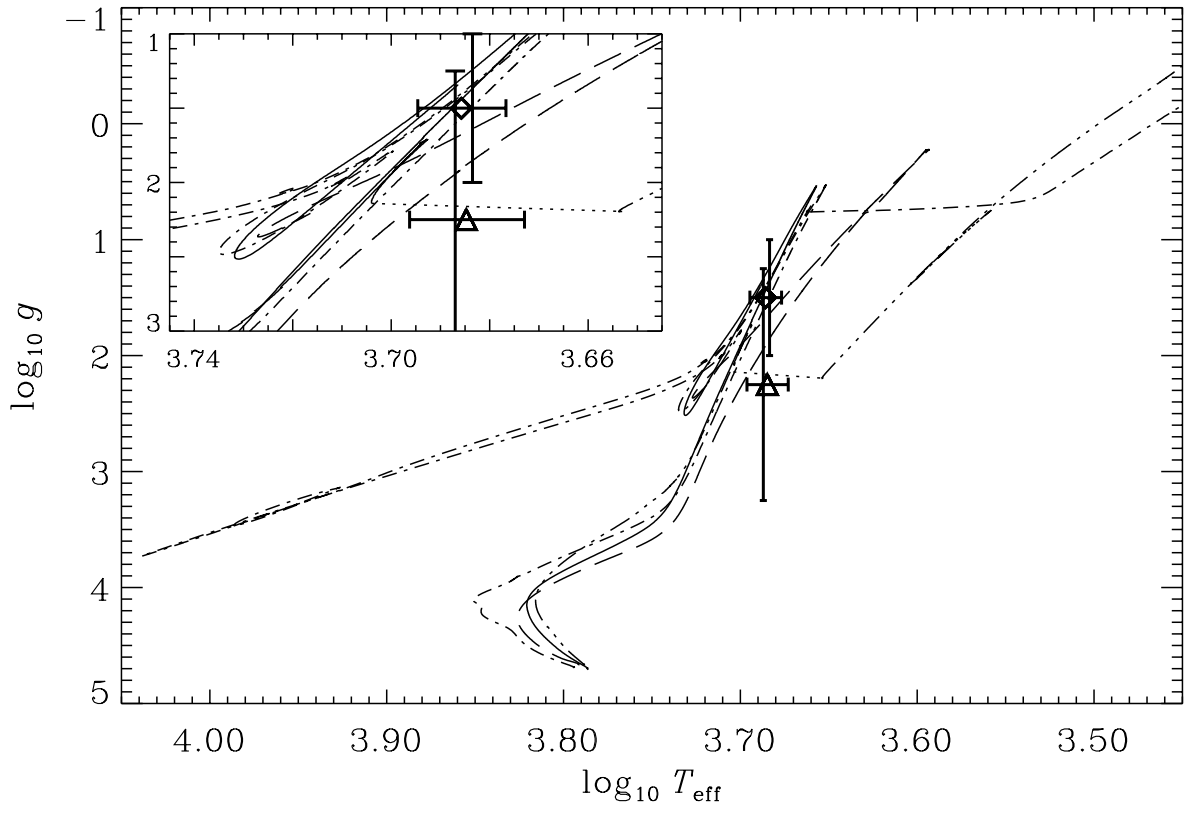

Fig. 1. Evolution in the gravity-effective temperature diagram of models with different initial metallicities: a $M=0.82 M_{\odot}$ models with initial $Z=0$ and surface metal pollution (dash-dot-dot-dotted line), a $0.78 M_{\odot}$-model with $Z=2.0 \times 10^{-5}$ ("best model", solid line), and a $0.82 M_{\odot}$-model with $Z=6.0 \times 10^{-4}$, where an initial C-, Nenhanced metal-mixture has been assumed (dashed line). In addition, a model with $Z=2.0 \times 10^{-5}$ and additional overshooting $(F=0.03)$ undergoing a HEFM during the TP-AGB phase is shown (dash-dotted line). Open symbols show the position of the two UMP stars CS 22892-052 and CS 22957027 (Table 1). The vertical error bars are slightly offset in order to not overlap. velocity obtained from a convection theory. We have discussed in Paper II that the convective velocities predicted by both mixing-length and Canuto \& Mazzitelli's (1991) theory lead to very similar results. In general, arbitrary changes by up to 2 orders of magnitude in the mixing speed do not affect appreciably the HEFM process.

Atomic diffusion is treated according to Thoul et al. (1994), opacities are from Iglesias \& Rogers (1996) and Alexander \& Ferguson (1994), neutrino energy losses from Munakata et al. (1985), and the equation of state (EOS) is a Saha EOS plus a simplified description of the degenerate electron gas in the core regions following Kippenhahn \& Weigert (1990). Our reaction network follows the abundances of $\mathrm{H},{ }^{3} \mathrm{He},{ }^{4} \mathrm{He},{ }^{12} \mathrm{C},{ }^{13} \mathrm{C},{ }^{14} \mathrm{~N}$, ${ }^{15} \mathrm{~N},{ }^{16} \mathrm{O},{ }^{17} \mathrm{O}$, without assuming a priori equilibrium compositions for any of these chemical elements. The reaction rates for the $p p$ - and CNO-burning have been taken from Adelberger et al. (1998), while the ${ }^{12} \mathrm{C}(\alpha, \gamma){ }^{16} \mathrm{O}$-rate agrees with Caughlan et al.'s (1985) value. The latter one is, in the relevant temperature range $\left(0.1<T /\left(10^{9} \mathrm{~K}\right)<0.2\right)$, about a factor of 2 higher than the rates of Caughlan \& Fowler (1988) and about 30-50\% higher than the most recent value of Kunz et al. (2002).

Figure 1, as a reference, displays the evolutionary track of the model with $0.82 M_{\odot}, Z=0$, and surface chemical pollution mentioned in the introduction (similar to model B1 of Paper II), which experiences the HEFM.

The $0.0003 M_{\odot}$ of $Z=0.02$ polluting material are instantaneously accreted before the star reaches the zero-age main sequence; this approach maximizes the effect of accretion and diffusion effects. After this episode the star evolves along the main sequence producing energy through the $p p$ chain. Diffusion is never able to bring the surface metals deep enough to contribute to the burning.

At the sub-giant branch the core increases its temperature and density, and gradually $3-\alpha$ reactions are setting in. In stars more massive than $\approx 0.9 M_{\odot}$ the central $\mathrm{H}$ content is not fully exhausted when sufficient $\mathrm{C}$ is produced $\left(X\left({ }^{12} \mathrm{C}\right) \approx 10^{-10}\right)$ to gain considerable nuclear energy from the $\mathrm{CNO}$-cycle. As a consequence a thermal runaway through the CNO-cycle ensues, causing a characteristic blue loop in the H-R diagram. The consequent expansion of the inner regions reduces the efficiency of the 3- $\alpha$ process and $\mathrm{CNO}-$ cycle, terminating the runaway which lasts only about $10^{7}$ years. In less massive models, as the ones considered in this work, the central $\mathrm{H}$ is already exhausted before sufficient $\mathrm{C}$ can be produced, and thus no blueloop occurs on the sub-giant branch.

Regardless of the occurrence of this CNO flash, all lowmass models settle on the RGB and evolve towards higher luminosities. While on the sub-giant branch their main energy source remains the $p p$-chain, the contribution of the CNOcycle is increasing gradually with luminosity as more carbon is created at the inner tail of the H-burning shell by 3- $\alpha$ reactions. In a $0.8 M_{\odot}$ star about $50 \%$ of the H-burning energy is produced by the CNO-cycle at the tip of the RGB.

At this stage the mass difference between the location of the maximum energy generation in the core and the He-core boundary is $\approx 0.29 M_{\odot}$. Soon after the onset of the He flash a convective shell develops, which reaches the H-rich matter in the envelope about 1 month after the flash (left arrow in Fig. 2); as soon as $\mathrm{H}$ is carried into the interior hotter convective region, it starts to burn at a very high rate. Due to this extra energy input the upper boundary of the convective shell immediately moves closer to the surface (thus ingesting even more protons), while the He-burning rate is significantly reduced. The single inner convective region splits into two zones, one for the $\mathrm{He}$ - and one for the H-burning. The rapidly weakening He-burning shell can no longer support the underlying layers against contraction, and the released gravothermal energy forms a further convective region which disappears again after a few 1000 years $^{1}$.

About $500 \mathrm{yr}$ after the $\mathrm{H}$ ingestion the convective envelope deepens and merges with the convective region above the

${ }^{1}$ This convective region appeared also in the calculations presented in Paper II, but was not shown in the corresponding figure due do insufficient plot data. 


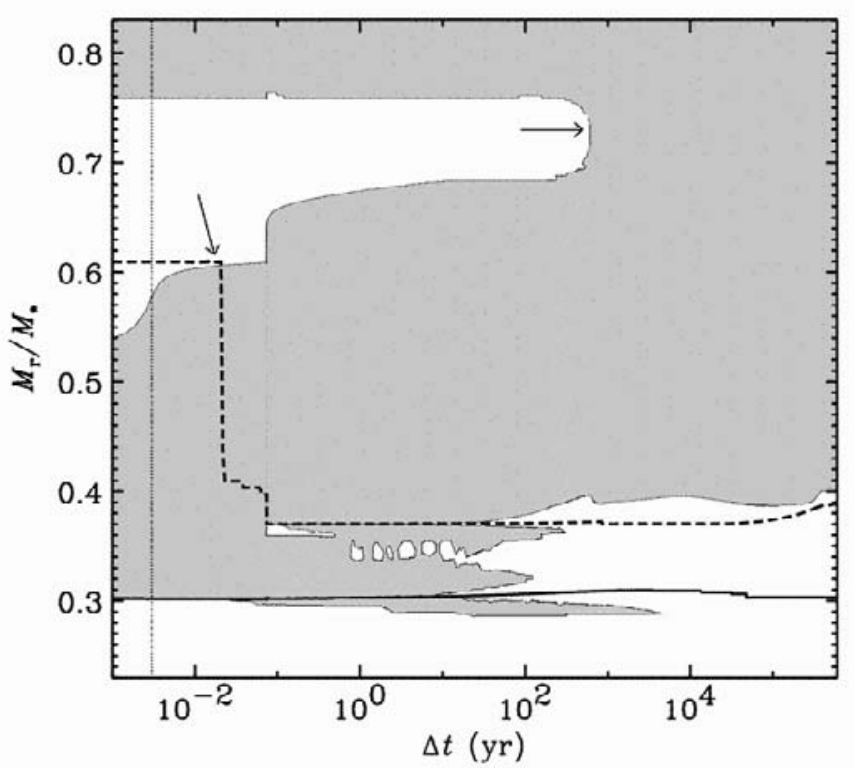

Fig. 2. Evolution of the different convective zones - indicated by shaded areas - from the He-flash ignition onwards in the $Z=0$ model of Fig. 1 with surface pollution. The dashed and solid lines represent the mass shells (in units of fraction of total mass) of maximum energy release by $\mathrm{H}$ and $\mathrm{He}$ burning, respectively. The two arrows mark the penetration of the He-flash driven convective zone into H-rich layers and the merger of the convective envelope with the $\mathrm{C}$ - and $\mathrm{N}$-rich inner convective region, respectively. The moment of maximum He-fusion energy release is indicated by the vertical dotted line. The zero point in time has been chosen to be 1 day before this moment. The shortliving small radiative layers between the shells are only marginally stable against convection ("semi-convection").

H-burning region (horizontal arrow in Fig. 2). A huge amount of matter processed through $\mathrm{H}$ and He burning is brought to the surface enriched in $\mathrm{He}, \mathrm{C}$ (produced during the He burning) and $\mathrm{N}$ (produced by the $\mathrm{CNO}$ cycle at the expenses of $\mathrm{C}$ ). The resulting surface $[\mathrm{C} / \mathrm{Fe}]$ and $[\mathrm{N} / \mathrm{Fe}]$ ratios are, respectively, 4.1 and 4.3 , while the surface oxygen abundance is practically unaltered with respect to the initial value (Table 2).

As a result of this dredge up of heavy elements (which happens on timescales of a few weeks) the envelope opacity is increased significantly; this produces an abrupt discontinuity in the effective temperature visible in Fig. 1. At this stage, with a practically ceased He burning, the star behaves as a newborn RGB star, climbing its own RGB. The H shell produces the energy needed to support the star. Another He flash ensues at the end of this second RGB phase, this time with a smaller and less degenerate core. The flash is weaker and no HEFM happens. The subsequent evolution does not show any peculiar feature.

As a general rule, all the changes in the physical inputs and/or initial conditions that can contribute to an increase of the electron degeneracy and thus cause the location of the He-flash ignition to move closer to the border of the He core favour the occurrence of HEFM. Changes in the relative location as small as $\approx 0.02 M_{\odot}$ can make a difference between models undergoing HEFM and models which do not experience it. In Paper II we have shown how an increase of the initial He abundance,

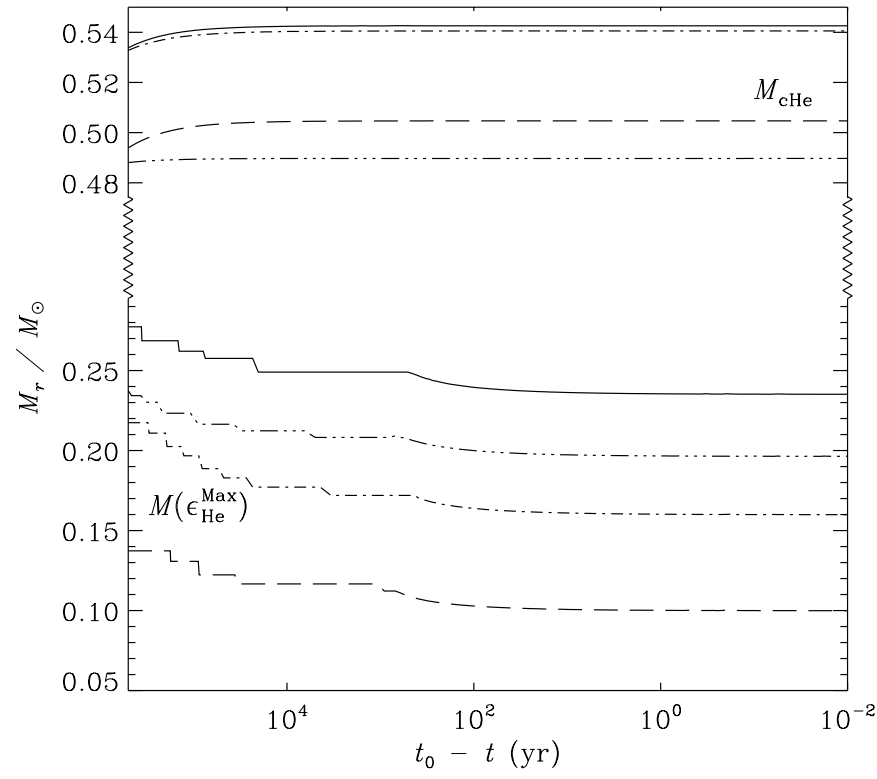

Fig. 3. Mass location of the He-core upper boundary and energy generation maximum as a function of the time before the flash $\left(t_{0}\right.$ indicates the onset of the flash), for the same models as in Fig. 1, indicated by the same line styles.

or an increase of the initial mass, or the inclusion of heavy element pollution at the stellar surface are disfavouring the onset of HEFM. On the other hand, the inclusion of element diffusion favours this process, while the inclusion of mass loss from the stellar surface does not affect at all the onset of the HEFM.

\section{The influence of initial metallicity and convection}

In Paper II we have investigated the dependence of the HEFM process on several input-physics parameters and various assumptions adopted in computing stellar models; however, we have considered only stars with initial zero metallicity, plus some eventual metal pollution at the surface. It is worthwhile to investigate the dependence of the HEFM on the value of the initial stellar metallicity, given the unknown composition of these stars at the time of formation, as outlined in the introduction.

Because of the uncertainty in the treatment of convection in stellar structures, it is also important to verify how much the HEFM process is sensitive to different assumptions about the extension and mixing efficiency of convective regions in the stellar interior. In particular, we considered various amounts of overshooting from the canonical formal convective boundaries fixed by the Schwarzschild criterion.

In this section we will discuss these effects in theoretical models representative of CS 22892-052 and CS 22957-027.

\subsection{Models with $Z>0$}

Models with initial $Y=0.23$ and increasing $Z$ have been computed in order to derive the value of the maximum initial metallicity which produces HEFM in a star with $0.82 M_{\odot}$. The heavy 
element mixture has been considered to be $\alpha$-enhanced, with $[\alpha / \mathrm{Fe}]=0.4$; carbon and nitrogen were scaled solar. We obtain HEFM only for $Z<10^{-7}$, corresponding to $[\mathrm{Fe} / \mathrm{H}]<$ -5.6 . Since all observed $[\mathrm{Fe} / \mathrm{H}]$-abundances in UMPs so far are higher than -4 , this already excludes the possibility that the $\mathrm{C} / \mathrm{N}$-anomalies were produced in stars with initial $\mathrm{Fe}$ abundances as observed today. Due to the mixing of H-rich material into the $\mathrm{H}$-depleted interior during the HEFM, the surface $\mathrm{H}$ abundance drops by about a factor of two, and the upper limit for the surface Fe abundance after this phase is $[\mathrm{Fe} / \mathrm{H}]<-5.3$, about 2 orders of magnitude smaller than the observed metallicities of CS 22892-052 and CS 22957-027. Whenever HEFM happens, the $[\mathrm{C} / \mathrm{Fe}]$ and $[\mathrm{N} / \mathrm{Fe}]$ ratios in the post He-flash phase are always of the order of 4 , still about 2 orders of magnitude higher than the observed values.

This upper value for the metallicity is slightly increased if we take into account the revised plasma-neutrino rates by Haft et al. (1994). In this case the limiting metallicity is $Z=10^{-6}$, corresponding to $[\mathrm{Fe} / \mathrm{H}]=-4.3$ after the HEFM, i.e., $1 \mathrm{dex}$ higher than in case of the old neutrino losses. The reason is that the degeneracy of the core increases and the He flash starts more off-centre. We also wish to notice here that, in spite of their different input physics and cruder treatment of the coupling between convection and burning, Fujimoto et al. (2000) found a similar upper limit for the metallicity of a $0.80 M_{\odot}$ star undergoing HEFM, and similar values of the surface $[\mathrm{C}, \mathrm{N} / \mathrm{Fe}]$ ratios.

We have also tested the effect of implementing a new EOS by Irwin (2000), which covers the entire stellar structure in all relevant evolutionary phases, and closely reproduces the OPAL EOS (Rogers et al. 1996) in the common validity range (see, e.g., Schlattl 2002). The inclusion of this new EOS in the models does not modify the value of the largest critical heavy elements abundance for which the HEFM occurs. However, with this equation of state the stellar ages are slightly reduced (by about $0.5 \mathrm{Gyr}$ ) with respect to our reference EOS, and therefore one has to choose a slightly lower mass to reproduce the observations, which might favour the occurrence of HEFM.

Therefore, we have computed a, what we call, "best model" with an initial $[\mathrm{Fe} / \mathrm{H}]$ equal to the observed one, and a mass which yields an age at the RGB tip well matching the age estimated for CS 22892-052 and CS 22957-027; we considered $M=0.78 M_{\odot}, Z=2.0 \times 10^{-5}$ (which corresponds, including the $\alpha$ enhancement by $0.4 \mathrm{dex}$, to $[\mathrm{Fe} / \mathrm{H}]=-3.3$ ), neutrino emission following Haft et al. (1994), Irwin's (2000) EOS, plus atomic diffusion of $\mathrm{H}, \mathrm{He}$, and metals (which further reduces the ages by about $0.5 \mathrm{Gyr}$ ). The corresponding evolutionary track is shown in Fig. 1, too (solid line). In spite of the reduced stellar mass the effect of the initial metallicity dominates, and no HEFM occurs. The location of the maximum energy generation at the flash is $0.30 M_{\odot}$ away from the boundary of the He core (solid line in Fig. 3), a distance about $0.01 M_{\odot}$ larger than in the cases when HEFM occurs. We computed also the evolution of stars of $0.75 M_{\odot}$ and $0.82 M_{\odot}$ with the same initial composition as the $0.78 M_{\odot}$ star to span the age range determined for CS 22892-052 from nuclear chronology, not obtaining HEFM in any case.

\subsection{Models including overshooting}

Models including overshooting have been computed in order to check its influence on both the onset of HEFM and the amount of $\mathrm{C}$ and $\mathrm{N}$ dredged up to the surface. Since overshooting increases the extension of convective regions, the occurrence of HEFM is in principle favoured. Our overshooting description follows Blöcker et al. (1998) and it is modelled as an exponential diffusive process. The diffusion constant of the overshoot region decays exponentially outside the Schwarzschild convective boundary, starting from the value assumed by the convective diffusion coefficient at the convective boundary; the decay length is $F$ times the pressure scale height at the convective boundary. Hydrodynamical simulations of shallow convective envelopes in A stars by Freytag et al. (1996) predict $F=0.25 \pm 0.05$, while for the overshooting from the convective envelopes of AGB stars Herwig et al. (1997) took $F=0.02$.

We have computed a series of models (adopting the same input physics as in Paper II) with different values for $F$, considering $M=0.82 M_{\odot}, Y=0.23$ and $Z=2.0 \times 10^{-5}$ which corresponds to $[\mathrm{Fe} / \mathrm{H}]=-3.3$, approximately the $[\mathrm{Fe} / \mathrm{H}]$ value determined for the two UMP stars under scrutiny. In these models the simpler EOS (Saha-EOS completed with degenerate electron gas in the deep interior) and the neutrino losses of Munakata et al. (1985) have been used.

We have first considered overshooting from all the convective boundaries, starting from the main-sequence phase. As a general result, we found that the inclusion of overshooting during the main sequence increases the lifetime of the small convective core which develops at the beginning of the main sequence, when the ${ }^{3} \mathrm{He}$ abundance gradually reaches nuclear equilibrium in the centre; this, in turn, causes an increase of the main-sequence lifetime and a decreased electron degeneracy in the He core along the RGB, which disfavours the occurrence of HEFM. Moreover, the increased lifetime would demand a larger stellar mass in order to obtain not too high stellar ages, and that would further reduce the probability for HEFM.

Models with $M=0.82 M_{\odot}$ and $F=0.03$ do not show HEFM, and are continuing their evolution through the horizontal branch to the TP-AGB (dash-dotted line in Fig. 1). During this phase the inter-shell convective zone is able to penetrate the H-rich envelope, similar to the HEFM process (Cassisi et al. 1996; Fujimoto et al. 2000). The surface $\mathrm{C}$ and $\mathrm{N}$ abundances rise again by about 4 dex, while $\mathrm{O}$ is increased by about 3 dex. Hence, an $\mathrm{O}$ enrichment about one order of magnitude smaller than for $\mathrm{C}$ is expected in these stars, which implies that $[\mathrm{O} / \mathrm{Fe}]$ should be at most 1 for the stars in Table 1. Since no oxygen abundance could be determined in these stars, but only an upper limit of $[\mathrm{O} / \mathrm{Fe}] \lessgtr 0.6$ (Sneden et al. 1996, for CS 22982-052), this scenario could provide a possible explanation for C-rich UMPs, too. However, surface gravity and temperature of these AGB stars are too low to be in agreement with the stars under scrutiny (Fig. 1).

Higher values of $F$ do not lead to HEFM. In fact, for $F=$ 0.1 there is no He flash, but quiescent He-burning ignition and of course no HEFM.

In case overshooting is included only from the RGB phase onwards, the stellar lifetime is not affected, and one needs at 


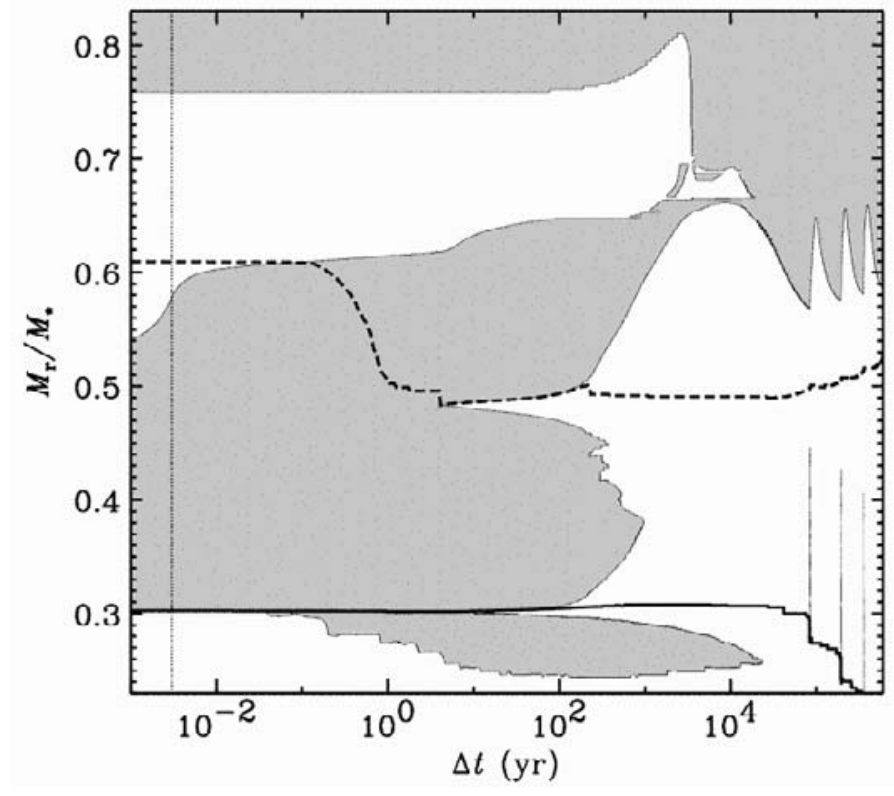

Fig. 4. Same model as in Fig. 1 but reducing the convective mixing efficiency by a factor $2 \times 10^{4}$.

least $F=0.17$ to get HEFM. This value is about $30 \%$ smaller as the one obtained by Freytag et al. (1996) for A-star envelopes, but appears to be quite high compared to that needed in AGB stars (Herwig et al. 1997); moreover, switching on overshooting only during the RGB phase appears to be entirely ad hoc; in addition, the resulting $[\mathrm{C} / \mathrm{Fe}]$ and $[\mathrm{N} / \mathrm{Fe}]$ ratios after the HEFM are again of the order of 4 , i.e., too high with respect to the observations.

\subsection{Models with reduced mixing efficiency}

From the computations performed until now, it appears that one of the main problem to the HEFM scenario is the too high $\mathrm{C}$ and $\mathrm{N}$ enrichment of the surface. One solution to obtain lower surface $[\mathrm{C}, \mathrm{N} / \mathrm{Fe}]$ ratios would be to have a smaller overlap between the convective region developing at the flash, and the overlying H-rich region. Smaller overlap implies less protons and therefore less processed $\mathrm{C}$ and $\mathrm{N}$. This smaller overlap could be achieved by strongly reducing the diffusion coefficient associated to the convective mixing. Using standard mixinglength velocities, the crossing time of the convective shell is of the order of a few hours. A reduction of the "convective" diffusion coefficient is performed by simply reducing the multiplicative factor that links the coefficient itself to the convective velocity.

The upper boundary of the He-flash driven convective shell is at first order dictated by the energy of the flash and the location of the ignition point. However, after some protons are engulfed the increased energy production (due to the additional $\mathrm{H}$ burning) pushes the upper boundary further up, thus increasing the number of proton ingested. If the mixing efficiency is reduced, protons are mixed less deep and less energy is produced, with a smaller increase of the convective shell extension. This would cause a smaller final number of protons ingested and a lower $\mathrm{N}$ production.
Table 2. Surface abundances of initial metal-free $0.82 M_{\odot}$ stars polluted by $3 \times 10^{-4} M_{\odot}$ of alpha-enhanced $\left.([\alpha / \mathrm{Fe}]=0.4]\right)$ material with $Z=0.02$ at the ZAMS. All models contain updated neutrino losses (Haft et al. 1994) and Irwin's (2000) EOS. The evolution of the convective regions after the flash are shown in the figures denoted in the first column. $f_{\mathrm{D}}$ is the factor by which the standard diffusion efficiency has been multiplied in each model.

\begin{tabular}{cccccccc}
\hline \hline Fig. & $f_{\mathrm{D}}$ & {$\left[\frac{\mathrm{C}}{\mathrm{Fe}}\right]$} & {$\left[\frac{\mathrm{N}}{\mathrm{Fe}}\right]$} & {$\left[\frac{\mathrm{O}}{\mathrm{Fe}}\right]$} & {$\left[\frac{\mathrm{Fe}}{\mathrm{H}}\right]$} & $\frac{{ }^{12} \mathrm{C}}{{ }^{13} \mathrm{C}}$ & $\frac{{ }^{13} \mathrm{C}}{{ }^{14} \mathrm{~N}}$ \\
\hline 2 & 1 & 4.1 & 4.3 & 0.7 & -3.4 & 4.5 & 0.36 \\
4 & $5 \times 10^{-5}$ & 2.3 & 3.2 & 0.4 & -3.4 & 5.6 & 0.07 \\
\hline
\end{tabular}

In order to explore this scenario in more detail, we recomputed the polluted $Z=0$ model of Sect. 2, which experiences HEFM, decreasing the "convective" diffusion coefficient by a factor of $2 \times 10^{4}$. The extension of the convective regions are displayed in Fig. 4. The upper boundary of the H-flash driven convective shell extends less into H-rich layers, and the location of the H-burning region is less deep; the thickness of the $\mathrm{H}$ convective shell before the dredge up is also thinner, implying a reduction of the amount of $\mathrm{C}$ dredged up with respect to the case with standard convective mixing. The final surface abundance ratios $[\mathrm{C} / \mathrm{Fe}]$ and $[\mathrm{N} / \mathrm{Fe}]$ are in this case 2.3 and 3.2 (Table 2), respectively, closer to the observed values.

The surface ${ }^{12} \mathrm{C} /{ }^{13} \mathrm{C}$ ratio is also affected, being now increased to 5.6 compared to 4.5 which we obtain by using the standard mixing efficiency. This increased value is in slightly better agreement with the observed values of about 10. In spite of the much reduced mixing efficiency the dredge up still happens fast, on timescales of the order of $10^{3}$ years.

\section{Discussion}

The results shown in the previous section highlight the fact that it is very difficult to reproduce the surface abundances of CS 22892-052 and CS 22957-027 from single-star evolution, at least in the case the observed surface abundances pattern is not primordial. To investigate this alternative, we have computed additionally the evolution of an $0.82 M_{\odot}$ star, with the same physics of our "best model" and $Y=0.23$, but with $Z=6 \times 10^{-4}$ and $[\mathrm{C} / \mathrm{Fe}]=[\mathrm{N} / \mathrm{Fe}]=2$ (implying $[\mathrm{Fe} / \mathrm{H}]=-3.3)$, in agreement with the observed abundances. The evolutionary track is shown in Fig. 1, while the He-core boundary before and at He ignition is plotted in Fig. 3. As expected, this model does not experience HEFM (the He flash starts very deep in the core), so that the surface $[\mathrm{C} / \mathrm{Fe}]$ and $[\mathrm{N} / \mathrm{Fe}]$ ratios are not modified with respect to the initial values. Note that the effective temperature along the RGB is higher than for the case with HEFM, because of the much lower Cabundance. The location of the track fits reasonably well the observed gravities and effective temperatures, and thus, this model would be able to reproduce the observed properties of CS 22957-027. However, as discussed in Sect. 1, if this were a realistic model for the observed object, the source for the high carbon and nitrogen abundances still remains unknown, as it is not clear how to pollute the interstellar medium with matter showing these anomalous abundance ratios. 
Thus, assuming that the observed surface composition is not primordial, the HEFM is presently the only way to get a surface $\mathrm{C}$ and $\mathrm{N}$ enhancement without invoking some kind of mass transfer from binary companions. From our tests in Sect. 2 there appear to be two main problems with the HEFM scenario:

Firstly, too much of $\mathrm{C}$ and $\mathrm{N}$ is transported to the surface after the HEFM, and secondly, no HEFM appears to occur for an initial metallicity that can match the observed $[\mathrm{Fe} / \mathrm{H}]$ of the stars under scrutiny, unless surface heavy element pollution has been efficient, as discussed in Paper II.

Once again, we want to point out that, in spite of their different input physics and cruder treatment of the coupling between convection and burning, Fujimoto et al. (2000) found similar upper limits for the metallicity of stars undergoing HEFM, and similar values of the $[\mathrm{C}, \mathrm{N} / \mathrm{Fe}]$ ratio for stellar mass and metallicity values similar to our "best model".

Concerning the appearance of HEFM in stars with $[\mathrm{Fe} / \mathrm{H}]$ in agreement with observations, one possible solution would be, as discussed in Sect. 3.3, a completely ad hoc choice of overshooting efficiency. Another possibility is related to the $\mathrm{H}$ depletion during the dredge up of the HEFM products. During this phase the convective envelope reaches deeper regions involved in the HEFM, which are devoid of $\mathrm{H}$. According to our models the surface $[\mathrm{Fe} / \mathrm{H}]$ increases by $\approx 0.3$ dex because of the dilution of $\mathrm{H}$. If the drop in hydrogen abundance is about five times higher, a surface $[\mathrm{Fe} / \mathrm{H}]$ of about -3.3 could be obtained with an initial $[\mathrm{Fe} / \mathrm{H}]=-4.3$ corresponding to the upper limit of getting HEFM.

In order to achieve a larger $\mathrm{H}$ depletion, a smaller envelope thickness (in mass) is needed, i.e., a smaller $\mathrm{H}$ reservoir. We estimate that right before the flash ignition an envelope of only about $0.03 M_{\odot}$ is needed in order to reproduce the observed post-HEFM $[\mathrm{Fe} / \mathrm{H}]$ surface values with our "best model"; with this envelope mass the star would still be able to ignite He close to its RGB location (see, e.g., Castellani \& Castellani 1993), i.e., the effective temperature and gravity after the HEFM would still be in accordance with CS 22892-052 and CS 22957-027. Taking into account the age of the star of about $14 \mathrm{Gyr}$, which fixes its initial mass to be about $0.8 M_{\odot}$, it would imply that about $0.27 M_{\odot}$ are lost during the evolution, a value which is not much higher than the average mass loss experienced by globular cluster stars (about $0.20 M_{\odot}$ ). The lack of metals in the outer layers of UMPs, however, should cause a strong decrease of mass-loss efficiency in comparison with more metal-rich stars, if radiative driven winds are the main source of mass loss. Nevertheless, due to our poor knowledge about mass-loss processes in RGB stars, this possibility cannot be completely ruled out.

Acknowledgements. We warmly thank A. Irwin for allowing us to use his new equation of state as well as for help in managing his routines. We also thank him for discussions and suggestions about the use of his EOS in the evolutionary code. We are grateful to T. Beers for his continuing interest and support of this work. One of us (S.C.) acknowledges the financial support by MURST/Cofin2000 under the project "Stellar Observables of Cosmological Relevance". H.S. has been supported by a Marie Curie Fellowship of the European Community programme "Human Potential" under contract number HPMF-CT2000-00951. A.W. thanks the Institute for Nuclear Theory at the University of Washington for its hospitality and the Department of Energy for partial support during the completion of this work.

\section{References}

Abel, T., Bryan, G. L., \& Norman, M. L. 2002, Science, 295, 93

Adelberger, E. G., Austin, S. M., Bahcall, J. N., et al. 1998, Rev. Mod. Phys., 70, 1265

Alexander, D. R., \& Ferguson, J. W. 1994, ApJ, 437, 879

Aoki, W., Norris, J. E., Ryan, S. G., Beers, T. C., \& Ando, H. 2002, ApJ, 567, 1166

Beers, T. C. 1999, in Connecting the Distant Universe with the Local Fossil Record, ed. M. Spite (Dordrecht: Kluwer Academic Publishers), Ap\&SS, 265, 547

Blöcker, T., Holweger, H., Freytag, B., et al. 1998, Space Sci. Rev., 85,105

Bonifacio, P., Molaro, P., Beers, T. C., \& Vladilo, G. 1998, A\&A, 332, 672

Bromm, V., Coppi, P. S., \& Larson, R. B. 2002, ApJ, 564, 23

Canuto, M., \& Mazzitelli, I. 1991, ApJ, 370, 295

Cassisi, S., Castellani, V., \& Tornambé, A. 1996, ApJ, 459, 298

Castellani, M., \& Castellani, V. 1993, ApJ, 407, 649

Caughlan, G. R., \& Fowler, W. A. 1988, Atomic Data Nuc. Data Tables, 40, 283

Caughlan, G. R., Fowler, W. A., Harris, M. J., \& Zimmerman, B. A. 1985, Atomic Data Nuc. Data Tables, 32, 197

Chieffi, A., Domínguez, I., Limongi, M., \& Straniero, O. 2001, ApJ, 554,1159

Cowan, J. J., Pfeiffer, S. G., Kratz, K.-L., et al. 1997, ApJ, 521, 194

Freytag, B., Ludwig, H.-G., \& Steffen, M. 1996, A\&A, 313, 497

Fujimoto, M. Y., Ikeda, Y., \& Iben, I. jr. 2000, ApJ, 529, L25

Haft, M., Raffelt, G., \& Weiss, A. 1994, ApJ, 425, 222

Heger, A., \& Woosley, S. E. 2002, ApJ, 567, 532

Herwig, F., Bloecker, T., Schoenberner, D., \& El Eid, M. 1997, A\&A, 324, L81

Iglesias, C. A., \& Rogers, F. J. 1996, ApJ, 464, 943

Irwin, A. W. 2000, GNU public license, FORTRAN routines available at ftp://astroftp. phys.uvic.ca/pub/irwin/eos/code

Kippenhahn, R., \& Weigert, A. 1990, Stellar Structure and Evolution (Berlin, Heidelberg: Springer-Verlag)

Kunz, R., Fey, M., Jaeger, M., et al. 2002, ApJ, 567, 643

Munakata, H., Kohyama, Y., \& Itoh, N. 1985, ApJ, 296, 197

Norris, J. E., Ryan, S. G., \& Beers, T. C. 1997a, ApJ, 488, 350

Norris, J. E., Ryan, S. G., \& Beers, T. C. 1997b, ApJ, 489, L169

Norris, J. E., Ryan, S. G., \& Beers, T. C. 2001, ApJ, 561, 1034

Preston, G. W., \& Sneden, C. 2001, ApJ, 122, 1545

Rogers, F. J., Swenson, F. J., \& Iglesias, C. A. 1996, ApJ, 456, 902

Rossi, S., Beers, T. C., \& Sneden, C. 1999, in The Third Stromlo Symp.: The Galactic Halo, ed. T. A. B. K. Gibson, \& M. Putma (San Francisco: ASP), ASP Conf. Ser., 165, 264

Schlattl, H. 2002, A\&A, 395, 85

Schlattl, S., Cassisi, S., Salaris, M., \& Weiss, A. 2001, ApJ, 559, 1082

Shigeyama, T., \& Tsujimoto, T. 1998, ApJ, 507, L135

Siess, L., Livio, M., \& Lattanzio, J. 2002, ApJ, 570, 329

Sneden, C., McWilliam, A., Preston, G. W., et al. 1996, ApJ, 467, 819

Thoul, A. A., Bahcall, J. N., \& Loeb, A. 1994, ApJ, 421, 828

Weiss, A., Cassisi, S., Schlattl, H., \& Salaris, M. 2000, ApJ, 533, 413

Weiss, A., \& Schlattl, H. 2000, A\&AS, 144, 487 